225

\section{T2-MAPPING CARDIAC MAGNETIC RESONANCE IMAGING FOR ASSESSING AREA-AT-RISK IN A MURINE MODEL OF MYOCARDIAL ISCHAEMIA-REPERFUSION INJURY}

R K Dongworth, ${ }^{1}$ A E Campbell-Washburn, ${ }^{2}$ T Roberts, ${ }^{2}$ D M Yellon, ${ }^{1}$ M F Lythgoe, ${ }^{2}$ DJ Hausenloy ${ }^{1}$ The Hatter Cardiovascular Institute, UCL; ${ }^{2}$ Centre for Advanced Biomedical Imaging, UCL

doi:10.1136/heartjnl-2013-304019.225

Rationale Novel therapeutic strategies are required to protect the heart against ischaemia-reperfusion injury. Assessment of the efficacy of cardioprotective strategies requires measurement of both myocardial infarct size (IS) and the area of 'at-risk' myocardium (AAR). Recent studies suggest that T2-weighted cardiac magnetic resonance imaging (cMR) can delineate AAR by detecting areas of myocardial oedema following ischaemia-reperfusion injury. However, applications of such cMR methods to the mouse heart are complicated by its small size and rapid heart rate. It was hypothesised that the use of T2-mapping $\mathrm{cMR}$ with improved resolution would permit accurate quantification of AAR in the mouse heart following ischaemia-reperfusion injury.

Methodology B6sv129 mice (10-12 weeks old) were subjected to an in vivo model of ischaemia-reperfusion injury comprising open chest surgery for LAD coronary artery ligation for 30 minutes followed by initiation of reperfusion and recovery of the animal. Characterisation of infarct size with varying periods of reperfusion ( $2 \mathrm{hrs}, 6 \mathrm{hrs}, 24$ hrs, $72 \mathrm{hrs}$ ) was first performed in a separate cohort of animals using histological staining with TTC and Evan's blue perfusion to determine AAR and infarct size by the 'gold-standard' method.

cMR Protocol Following 3 days reperfusion, mice underwent cMR briefly consisting of late-gadolinium enhancement and T2-mapping imaging for the assessment of infarct size and putative AAR respectively. Immediately following $\mathrm{cMR}$, mice were anaesthetised and hearts extracted and subjected to classical histological staining as described above. Assessment of cMR quantifications was performed by comparison with equivalent histological staining of left ventricular myocardial slices for infarct size (IS/LV\%) and AAR (AAR/LV\%).
Results Our initial characterisation study demonstrated no significant change in infarct size over the initial $72 \mathrm{hrs}$ reperfusion examined. cMR late-gadolinium enhancement imaging for the quantification of infarct size demonstrated excellent agreement with histological staining (IS/LV\%: cMR late-Gd 30.90 0.6 ; histology $30.72 \pm 2.6: n=5, p=0.95)$. Importantly cMR T2-maps of the myocardium showed clearly defined regions of elevated T2-signal in spatially distinct regions of the myocardium. Comparison of T2-maps and histological staining for each myocardial slice demonstrated a good agreement in the spatial localisation of AAR judged by T2-cMR and histology. Quantification of AAR from these T2-maps showed good agreement with AAR quantified byhistological staining (AAR/LV\%: T2-cMR 62.10 44.1; histology 64.78 $\pm 6.5: n=6, p=0.55)$.

Conclusions The T2-mapping cMR protocol developed in the present study permits accurate quantification of myocardial area-at-risk in mice following ischaemia-reperfusion injury. As such, this $\mathrm{cMR}$ method provides a potential tool for the assessment of myocardial salvage in future studies of cardioprotective efficacy in the setting of ischaemia-reperfusion injury. 